\title{
Some exact results and new asymptotics for hypergraph Turán numbers
}

\author{
Dhruv Mubayi *
}

June 11, 2005

\begin{abstract}
Given a family $\mathcal{F}$ of $r$-graphs, let $\operatorname{ex}(n, \mathcal{F})$ be the maximum number of edges in an $n$ vertex $r$-graph containing no member of $\mathcal{F}$. Let $C_{4}^{(r)}$ denote the family of $r$-graphs with distinct edges $A, B, C, D$, such that $A \cap B=C \cap D=\emptyset, A \cup B=C \cup D$. For $s_{1} \leq \cdots \leq s_{r}$, let $K^{(r)}\left(s_{1}, \ldots, s_{r}\right)$ be the complete $r$-partite $r$-graph with parts of sizes $s_{1}, \ldots, s_{r}$.
\end{abstract}

Füredi conjectured over 15 years ago that $\operatorname{ex}\left(n, C_{4}^{(3)}\right) \leq\left(\begin{array}{c}n \\ 2\end{array}\right)$ for $n$ sufficiently large. We prove the weaker result $\operatorname{ex}\left(n,\left\{C_{4}^{(3)}, K^{(3)}(1,2,4)\right\}\right) \leq\left(\begin{array}{l}n \\ 2\end{array}\right)$.

Generalizing a well-known conjecture for the Turán number of bipartite graphs, we conjecture that

$$
\operatorname{ex}\left(n, K^{(r)}\left(s_{1}, \ldots, s_{r}\right)\right)=\Theta\left(n^{r-1 / s}\right),
$$

where $s=\prod_{i=1}^{r-1} s_{i}$. We prove this conjecture when $s_{1}=\cdots=s_{r-2}=1$ and (i) $s_{r-1}=2$, (ii) $s_{r-1}=s_{r}=3$, (iii) $s_{r}>\left(s_{r-1}-1\right)$ !. In cases (i) and (ii), we determine the asymptotic value of $\operatorname{ex}\left(n, K^{(r)}\left(s_{1}, \ldots, s_{r}\right)\right)$.

We also provide an explicit construction giving

$$
\operatorname{ex}\left(n, K^{(3)}(2,2,3)\right)>(1 / 6-o(1)) n^{8 / 3} .
$$

This improves upon the previous best lower bound of $\Omega\left(n^{29 / 11}\right)$ obtained by probabilistic methods. Several related open problems are also presented.

*School of Mathematics, Georgia Institute of Technology, Atlanta, GA 30332, mubayi@math.gatech.edu Research supported in part by the National Science Foundation under grant DMS-9970325 1991 Mathematics Subject Classification: 05B05, 05B07, 05B25, 05C35, 05C80, 05D05, 11 T99

Keywords: Turán numbers, r-partite hypergraphs, algebraic constructions 


\section{Introduction}

Given a family of $r$-uniform hypergraphs (or $r$-graphs) $\mathcal{F}$, we say that an $r$-graph $\mathcal{G}$ is $\mathcal{F}$ free if $\mathcal{G}$ contains no subhypergraph isomorphic to any element in $\mathcal{F}$. Let $\operatorname{ex}(n, \mathcal{F})$ be the maximum number of edges in an $n$ vertex $\mathcal{F}$-free $r$-graph. If $\mathcal{F}=\left\{K_{k}^{(r)}\right\}$, the complete $r$-graph on $k$ vertices, then $\operatorname{ex}(n, \mathcal{F})$ is the Turán number $t_{r}(n, k)$. The determination of $\lim _{n \rightarrow \infty} t_{r}(n, k) /\left(\begin{array}{l}n \\ r\end{array}\right)$ is perhaps the most fundamental open problem in extremal hypergraph theory. We consider the related question of determining ex $(n, \mathcal{F})$ when $\mathcal{F} \neq\left\{K_{k}^{(r)}\right\}$.

Definition 1.1. Let $C_{4}^{(r)}$ be the family of r-graphs consisting of distinct edges $A, B, C, D$ with $A \cap B=C \cap D=\emptyset$ and $A \cup B=C \cup D$. For $1 \leq s_{1} \leq \cdots \leq s_{r}$, let $K^{(r)}\left(s_{1}, \ldots, s_{r}\right)$ be the $r$-partite $r$-graph with vertex set consisting of disjoint sets $X_{1}, \ldots, X_{r}$ of sizes $s_{1}, \ldots, s_{r}$, respectively, and all edges of the form $\left\{x_{1}, \ldots, x_{r}\right\}$, where $x_{i} \in X_{i}$.

When $r=3, C_{4}^{(r)}$ consists of only one isomorphism class of 3-graphs, and so we sometimes abuse notation by referring to this 3-graph as $C_{4}^{(3)}$. Let $f_{r}(n)=\operatorname{ex}\left(n, C_{4}^{(r)}\right)$. Determining $f_{2}(n)$ is a well-known graph problem that goes back to 1938 [8]. For general $r$, this function was introduced by Erdős in 1977 [4]. Füredi [12] proved the following result.

Theorem 1.2. (Füredi [12]) If $r \geq 3$, then $\left(\begin{array}{c}n-1 \\ r-1\end{array}\right)+\left\lfloor\frac{n-1}{r}\right\rfloor \leq f_{r}(n)<3.5\left(\begin{array}{c}n \\ r-1\end{array}\right)$. Also

$$
f_{3}(n) \geq\left(\begin{array}{l}
n \\
2
\end{array}\right) \text { for } n \equiv 1,5(\bmod 20)
$$

Conjecture 1.3. (Füredi [12]) If $r \geq 3$, and $n>n_{0}(r)$, then $f_{r}(n) \leq\left(\begin{array}{c}n \\ r-1\end{array}\right)$. Moreover, for $r \geq 4$, we have $f_{r}(n)=\left(\begin{array}{l}n-1 \\ r-1\end{array}\right)+\left\lfloor\frac{n-1}{r}\right\rfloor$.

Although we are unable to prove Conjecture 1.3, we provide some additional evidence for its truth when $r=3$ by proving

Theorem 1.4. $\operatorname{ex}\left(n,\left\{C_{4}^{(3)}, K^{(3)}(1,2,4)\right\}\right) \leq\left(\begin{array}{l}n \\ 2\end{array}\right)$.

Füredi's example that yields (1) is obtained by first taking a Steiner system of 5-sets (i.e., an $n$ vertex 5-graph in which every pair of vertices is contained in precisely one edge), and then replacing each 5 -set by the ten triples contained in it. This yields a 3-graph with $\left(\begin{array}{l}n \\ 2\end{array}\right)$ edges. It is well-known [20] that such Steiner systems exist when $n \equiv 1,5(\bmod 20)$. 
Moreover, it is easy to see that this construction is both $C_{4}^{(3)}$-free and $K^{(3)}(1,2,4)$-free, and hence Theorem 1.4 is sharp.

While the above result is admittedly modest, we hope that it fosters some new ideas towards the proof of Conjecture 1.3, at least for $r=3$, and also leads to new problems.

It is a well-known open graph problem to determine the growth rate of $\operatorname{ex}\left(n, K_{a, b}\right)$ for fixed $a \leq b$. The classical upper bound due to Kövári, Sós and Turán [18] gives for $1 \leq a \leq b$,

$$
2 \operatorname{ex}\left(n, K_{a, b}\right) \leq(b-1)^{1 / a} n^{2-(1 / a)}+(a-1) n .
$$

These are conjectured to be asymptotically optimal as $n \rightarrow \infty$ for every fixed $a \leq b$. This has been proved for $b \geq a=2$ (Brown [6], Erdős, Rényi, Sós [10], and Füredi [13]), $a=b=3$ [6, 14], and for every $a>3$ and $b>(a-1)$ ! by Alon, Rónyai, and Szabó [2]. The best known general bound, obtained by the probabilistic method (see, e.g., [5], p. 310, or [11], p. 61, proof of inequality $(12.19)$ ), is

$$
\operatorname{ex}\left(n, K_{a, b}\right) \geq \Omega\left(n^{2-\frac{a+b-2}{a b-1}}\right)
$$

In light of Theorem 1.4, it seems appropriate to promote the study of Turán numbers for 3-partite 3-graphs, i.e., the numbers $\operatorname{ex}\left(n, K^{(3)}(i, j, k)\right)$. An old result of Erdös [9] implies that $\operatorname{ex}\left(n, K^{(3)}(i, j, k)\right) \leq O\left(n^{3-1 / i j}\right)$, and when $i=j=k$, Erdös indicates in [9] how to prove a lower bound of $\Omega\left(n^{3-C / i j}\right)$ for an absolute constant $C$. Erdös also remarks in [9] that $\operatorname{ex}\left(n, K^{(3)}(l, l, l)\right)=\Theta\left(n^{3-1 / l^{2}}\right)$ could hold, but this has not been proven for any $l>1$. In fact, it appears that even $\operatorname{ex}\left(n, K^{(3)}(i, j, k)\right)=\Theta\left(n^{3-1 / i j}\right)$ was not known for any $j>1$. We prove this for $i=1$ and

(a) $k \geq j=2$,

(b) $j=k=3$,

(c) $j>3, k \geq(j-1) !+1$.

For (a) and (b) we also determine the asymptotics. We also extend these results to $r$-graphs. Our constructions are very slight variations of Füredi's algebraic construction from [13] and the constructions of Alon et. al. in [2] and yield, for example,

$$
\operatorname{ex}\left(n, K^{(3)}(1,2,4)\right) \sim\left(\frac{1}{2 \sqrt{3}}\right) n^{5 / 2} .
$$

Note that (2) also implies that Theorem 1.4 is not vacuous by showing that far more than $\left(\begin{array}{l}n \\ 2\end{array}\right)$ edges are required to force a copy of just $K^{(3)}(1,2,4)$. 
Based on these extensions of graph constructions to hypergraphs, we make the following conjecture which is probably widely believed but appears never to have been stated in such generality.

Conjecture 1.5. Let $r>1, s_{1} \leq \cdots \leq s_{r}$ be fixed. Then

$$
\operatorname{ex}\left(n, K^{(r)}\left(s_{1}, \ldots, s_{r}\right)\right)=\Theta\left(n^{r-1 / s}\right),
$$

where $s=\prod_{i=1}^{r-1} s_{i}$.

In addition to the results mentioned above, we improve the previous best known lower bounds for another isolated case.

\section{Theorem 1.6.}

$$
\operatorname{ex}\left(n, K^{(3)}(2,2,3)\right)>(1 / 6-o(1)) n^{8 / 3} .
$$

It is mentioned in [17] that it would be useful to obtain explicit constructions of large graphs without $K_{t, t}$ even if the density is far worse than that guaranteed by probabilistic constructions. Such constructions would be useful in computing theory [1]. The best known lower bound $\operatorname{ex}\left(n, K^{(3)}(2,2,3)\right)>\Omega\left(n^{29 / 11}\right)$ is obtained by probabilistic methods. Theorem 1.6 improves this by an explicit construction.

In section 2 , we present the proof of Theorem 1.4, and a related probabilistic result. In sections 3 and 4 we prove the results on Turán numbers for $r$-partite $r$-graphs. In section 5 we prove Theorem 1.6. Related problems and conjectures are discussed in section 6 .

Throughout we denote the vertex set and edge set of a hypergraph $\mathcal{H}$ by $V(\mathcal{H})$ and $E(\mathcal{H})$, respectively. The degree of a vertex $v$ in $\mathcal{H}$ is the number of edges containing $v$; the neighborhood of $v$ is the set of vertices $v^{\prime}$ for which there is an edge $S \in E(\mathcal{H})$ such that $\left\{v, v^{\prime}\right\} \subseteq S$. Given functions $f$ and $g, f=O(g)$ means that there is an absolute constant $c$ such that for $n$ sufficiently large, $|f(n) / g(n)|<c ; f=\Omega(g)$ if $g=O(f)$, and $f=o(g)$ means that $\lim _{n \rightarrow \infty} f(n) / g(n)=0$. We say that $f$ is asymptotic to $g$ if $\lim _{n \rightarrow \infty} f(n) / g(n)=1$, and we write $f \sim g$. Equivalently, $f \sim g$ if and only if $f=(1+o(1)) g$. If $f=O(g)$ and $g=O(f)$, then we write $f=\Theta(g)$.

\section{Generalized four-cycles}

By an $r$-star we mean a star with $r$ edges. Our main tool is to count the number of $r$-stars in bipartite graphs. The following Lemma is a special case of results in [7, 19], we present it 
so that our proof is self-contained.

Lemma 2.1. Let $r \geq 2$ be an integer. Let $G$ be a bipartite graph with parts $X, Y$, of sizes $\left(\begin{array}{c}n \\ r-1\end{array}\right)$ and $n$, respectively. If $|E(G)|>r\left(\begin{array}{c}n \\ r-1\end{array}\right)$, then $G$ contains a copy of $K_{r+1, r-1}$ with $r+1$ vertices in $X$ and $r-1$ vertices in $Y$.

Proof. Let $p$ be the number of $(r-1)$-stars in $G$ with leaves in $Y$. By the convexity of binomial coefficients, we get

$$
p=\sum_{x \in X}\left(\begin{array}{c}
d(x) \\
r-1
\end{array}\right)>\left(\begin{array}{c}
n \\
r-1
\end{array}\right)\left(\begin{array}{c}
r \\
r-1
\end{array}\right)=r\left(\begin{array}{c}
n \\
r-1
\end{array}\right) .
$$

Hence at least $r+1$ distinct $(r-1)$-stars share the same $(r-1)$-set of leaves in $Y$.

Definition 2.2. Fix $r \geq 3$. Let $\mathcal{H}^{(r)}$ be the family of $r$-graphs with vertex set consisting of disjoint sets $X \cup Y$, where $X=X_{1} \cup X_{2},\left|X_{1}\right|=\left|X_{2}\right|=r-1,\left|X_{1} \cap X_{2}\right| \leq r-3,|Y|=r-1$, and edge set $\left\{A \cup y: A \in\left\{X_{1}, X_{2}\right\}, y \in Y\right\}$.

We prove Theorem 1.4 in a slightly more general form. Given a set $S$ and a positive integer $k$, we let $\left(\begin{array}{l}S \\ k\end{array}\right)$ denote the family of $k$-subsets of $S$.

Theorem 2.3. Let $r \geq 3$. Then $\operatorname{ex}\left(n,\left\{\mathcal{H}^{(r)}, K^{(r)}(1, \ldots, 1, r-1, r+1)\right\}\right) \leq\left(\begin{array}{c}n \\ r-1\end{array}\right)$.

Proof. Let $\mathcal{G}$ be an $n$ vertex $r$-graph with vertex set $V$ and $e>\left(\begin{array}{c}n \\ r-1\end{array}\right)$ edges. Form the bipartite graph $B$ with parts $X=\left(\begin{array}{c}V \\ r-1\end{array}\right)$ and $Y=V$, and edges $(S, y)$, where $S \cup y$ is an edge of $\mathcal{G}$.

Since each edge of $\mathcal{G}$ is counted $r$ times in $B$, we have $|E(B)|>r\left(\begin{array}{c}n \\ r-1\end{array}\right)$. Applying Lemma 2.1 yields a copy $H$ of $K_{r+1, r-1}$ in $B$. It remains only to show that $H$ corresponds to either $\mathcal{H}^{(r)}$ or $K^{(r)}(1, \ldots, 1, r-1, r+1)$ in $\mathcal{G}$.

Let $V_{X}=V(H) \cap X$ and $V_{Y}=V(H) \cap Y$. If there exist $S, T \in V_{X}$ with $|S \cap T| \leq r-3$, then the edge set $\left\{A \cup y: A \in\{S, T\}, y \in V_{Y}\right\}$ forms a copy of $\mathcal{H}^{(r)}$ in $\mathcal{G}$.

Hence we may assume that for every pair of distinct elements $S, T$ in $V_{X}$, we have $|S \cap T|=$ $r-2$. This implies that the $r+1$ sets in $V_{X}$ form a sunflower with kernel of size $r-2$. These $(r-1)$-sets form a copy of $K^{(r-1)}(1, \ldots, 1, r+1)$, where the $r-2$ parts of size one correspond to the elements of the kernel. It is now easy to see that the $r$-sets defined by $H$ form a copy of $K^{(r)}(1, \ldots, 1, r-1, r+1)$ in $\mathcal{G}$. 
Since $\mathcal{H}^{(3)}$ is the family $C_{4}^{(3)}$, the special case $r=3$ of Theorem 2.3 is precisely Theorem 1.4. We next prove a straightforward generalization of a well-known result for graphs.

Lemma 2.4. Suppose that $\mathcal{H}$ is an r-graph with $v$ vertices and e edges. Then $\operatorname{ex}(n, \mathcal{H}) \geq$ $\Omega\left(n^{r-v / e}\right)$. Moreover, almost every $n$ vertex $r$-graph with o( $\left.n^{r-v / e}\right)$ edges is $\mathcal{H}$-free.

Proof. The proof mirrors [3], pages 41-42, and is an easy application of the first moment method. Consider the probability space of all $n$ vertex $r$-graphs on $m$ edges, where each $r$-graph with $m$ edges is equally likely. Let $\mathcal{G}$ be an element of this probability space. For each $S \subseteq V(\mathcal{G})$ with $|S|=v$, let $A_{S}$ be the event that the subhypergraph induced by $S$ contains a copy of $\mathcal{H}$. Then

$$
\operatorname{Pr}(\mathcal{G} \text { is not } \mathcal{H} \text {-free })=\operatorname{Pr}\left(\bigcup_{S} A_{S}\right) \leq O\left(\left(\begin{array}{c}
n \\
v
\end{array}\right) \frac{\left(\begin{array}{c}
n \\
r \\
m-e
\end{array}\right)}{\left(\begin{array}{c}
n \\
r \\
m
\end{array}\right)}\right)=O\left(m^{e} n^{v-r e}\right) .
$$

If $m=o\left(n^{r-v / e}\right)$, then $\operatorname{Pr}\left(\bigcup_{S} A_{S}\right)=o(1)$. If $c$ is a sufficiently small constant, and $m=$ $c n^{r-v / e}$, then $\operatorname{Pr}\left(\bigcup_{S} A_{S}\right)<1$.

Conjecture 1.3 for $r=3$ holds for almost all 3-graphs on $\left(\begin{array}{l}n \\ 2\end{array}\right)$ edges, but a direct proof requires using the second moment. Theorem 1.4 provides a simple first moment proof of this fact, since it suffices to forbid $K^{(3)}(1,2,4)$. Indeed, together with Lemma 2.4 , it easily implies

Corollary 2.5. Almost all n vertex 3-graphs with $\left(\begin{array}{l}n \\ 2\end{array}\right)+1$ edges are not $C_{4}^{(3)}$-free.

\section{Asymptotics for $\operatorname{ex}\left(n, K^{(r)}(1, \ldots, 1,2, t+1)\right)$}

In this section we determine the asymptotics for an infinite class of Turán numbers for $r$ partite $r$-graphs using the construction developed in [13]. Since the construction and many of the ideas are identical with those in [13], we emphasize only the new points.

Theorem 3.1. Let $r, t$ be fixed positive integers, $r \geq 2$. Let $\mathcal{H}$ be the complete $r$-partite $r$-graph $K^{(r)}(1, \ldots, 1,2, t+1)$ consisting of $r-2$ sets of size 1 , one set of size 2, and one set of size $t+1$. Then

$$
\operatorname{ex}(n, \mathcal{H}) \sim \frac{\sqrt{t}}{r !} n^{r-1 / 2}
$$


Proof. We first give a construction that proves the lower bound. Let $q$ be a prime power such that $(q-1) / t$ is an integer, and let $n=(q-1)^{2} / t$. We define an $n$ vertex $\mathcal{H}$-free $r$-graph $\mathcal{G}$ with at least

$$
\frac{q^{2 r-1}}{t^{r-1} r !}-O\left(q^{2 r-2}\right)
$$

edges. Then the lower bound for the Turán number for all $n$ follows from the fact that for every sufficiently large $n$ there exists a prime $q$ satisfying $q \equiv 1(\bmod t)$ and $\sqrt{n t}-n^{1 / 3}<$ $q<\sqrt{n t}($ see $[16])$

Let $\mathbf{F}$ be the $q$-element finite field, and let $H$ be a $t$-element subgroup of $\mathbf{F} \backslash\{0\}$. The elements of the vertex set $V$ consist of equivalence classes in $(\mathbf{F} \backslash\{0\}) \times(\mathbf{F} \backslash\{0\})$, where $(a, b) \sim(x, y)$ if there is an $\alpha \in H$ such that $a=\alpha x$ and $b=\alpha y$. The class represented by $(a, b)$ is denoted by $\langle a, b\rangle$. A set of $r$ distinct classes $\left\langle a_{i}, b_{i}\right\rangle(1 \leq i \leq r)$ form an edge in $\mathcal{G}$ if

$$
\prod_{i=1}^{r} a_{i}+\prod_{i=1}^{r} b_{i} \in H
$$

It is easily observed that this relation is well-defined, and that the number of edges in $\mathcal{G}$ is at least

$$
\left(\begin{array}{c}
n \\
r-1
\end{array}\right) \frac{q-r}{r}=\left(\begin{array}{c}
(q-1)^{2} / t \\
r-1
\end{array}\right) \frac{q-r}{r}=\frac{q^{2 r-1}}{t^{r-1} r !}-O\left(q^{2 r-2}\right),
$$

as required in (4).

Next we show that $\mathcal{G}$ is $\mathcal{H}$-free. For $(a, b),\left(a^{\prime}, b^{\prime}\right) \in(\mathbf{F} \backslash\{0\}) \times(\mathbf{F} \backslash\{0\}),(a, b) \nsim\left(a^{\prime}, b^{\prime}\right)$ the equation system

$$
\begin{array}{r}
a x+b y=\alpha \\
a^{\prime} x+b^{\prime} y=\beta
\end{array}
$$

has at most one solution $(x, y)$ for every $\alpha, \beta \in H$. From this it follows that the system

$$
\begin{gathered}
a x+b y \in H \\
a^{\prime} x+b^{\prime} y \in H
\end{gathered}
$$

has at most $t$ nonequivalent solutions $(x, y)$. See [13] for the details.

Now suppose that $\left\langle a_{i}, b_{i}\right\rangle, 1 \leq i \leq r-2,\left\langle u_{j}, v_{j}\right\rangle, 1 \leq j \leq 2,\left\langle x_{k}, y_{k}\right\rangle, 1 \leq k \leq t+1$, form the vertex set of a copy of $\mathcal{H}$ in $\mathcal{G}$, where the $\left\langle a_{i}, b_{i}\right\rangle$ form the parts of size $1,\left\{\left\langle u_{j}, v_{j}\right\rangle\right\}$ forms 
the part of size 2 , and $\left\{\left\langle x_{k}, y_{k}\right\rangle\right\}$ forms the part of size $t$. For $j=1,2$, set

$$
p_{j}=\left(\prod_{i=1}^{r-2} a_{i}\right) u_{j} \quad \text { and } \quad q_{j}=\left(\prod_{i=1}^{r-2} b_{i}\right) v_{j} .
$$

Note that $\left(p_{1}, q_{1}\right) \nsim\left(p_{2}, q_{2}\right)$, since otherwise $\left\langle u_{1}, v_{1}\right\rangle=\left\langle u_{2}, v_{2}\right\rangle$. The edges forming the copy of $\mathcal{H}$ yield, for $1 \leq k \leq t+1$,

$$
\begin{aligned}
& p_{1} x_{k}+q_{1} y_{k} \in H \\
& p_{2} x_{k}+q_{2} y_{k} \in H
\end{aligned}
$$

where $\left\langle x_{l}, y_{l}\right\rangle \neq\left\langle x_{l}^{\prime}, y_{l}^{\prime}\right\rangle$ when $l \neq l^{\prime}$. But we have argued in (7) that such a system can have at most $t$ nonequivalent solutions. This completes the proof of the lower bound.

For the upper bound, we proceed by induction on $r$. The case $r=2$ is proved in [18]. The inductive step follows immediately by considering the $(r-1)$-graph induced by the neighborhood of a vertex of maximum degree.

\section{Asymptotics for $\operatorname{ex}\left(n, K^{(r)}(1, \ldots, 1,3,3)\right)$}

In [17] an algebraic construction is given which proves that $\operatorname{ex}\left(n, K_{s, t}\right)=\Theta\left(n^{2-1 / s}\right)$ for $t \geq s !+1$. This construction is extended to $t \geq(s-1) !+1(s \geq 3)$ in [2]. In this section, we show that a very slight variation of this construction yields the correct order of magnitude for $\operatorname{ex}\left(n, K^{(r)}(1, \ldots, 1, s, t)\right.$ ) (for $s \geq 3, t=(s-1) !+1$ ); it also yields the asymptotic value of $\operatorname{ex}\left(n, K^{(r)}(1, \ldots, 1,3,3)\right)$. The extension to hypergraphs is much the same as the extension of Füredi's construction in the previous section.

We now define the "Norm-hypergraph" $\mathcal{G}=\mathcal{G}^{(r)}(q, s)$ for any $s>2$. Let $V(\mathcal{G})=$ $G F\left(q^{s-1}\right) \times G F(q)^{*}$, where $G F(p)$ is the finite field of $p$ elements, and $G F(p)^{*}=G F(p) \backslash\{0\}$. The vertices $\left(A_{i}, a_{i}\right), i=1, \ldots, r$ form an edge if

$$
N\left(\sum_{i=1}^{r} A_{i}\right)=\prod_{i=1}^{r} a_{i}
$$

where $N(X)=X^{1+q+\ldots+q^{s-2}}$ is the norm of $X \in G F\left(q^{s-1}\right)$ over $G F(q)$. Note that for $X \in G F\left(q^{s-1}\right)$, we have $(N(X))^{q}=N(X)$, so $N(X) \in G F(q)$ (indeed, $G F(q)$ consists of precisely the solutions to $\left.x^{q}-x=0\right)$. For every choice of $r-1$ vertices $\left(A_{i}, a_{i}\right), 1 \leq i \leq r-1$, 
there are $q^{s-1}-r$ pairs $(A, a), A \notin\left\{A_{1}, \ldots, A_{r-1},-\sum_{i} A_{i}\right\}$, which satisfy (8). Since each edge is counted at most $r$ times in this way the number of edges in $\mathcal{G}$ is at least

$$
\left(\begin{array}{c}
n \\
r-1
\end{array}\right) \frac{q^{s-1}-r}{r}=\left(\begin{array}{c}
q^{s}-q^{s-1} \\
r-1
\end{array}\right) \frac{q^{s-1}-r}{r}=\frac{q^{r s-1}}{r !}-O\left(q^{r s-2}\right) .
$$

We need the following result that is proved in [2] but follows easily from a result in [17] Lemma 4.1. If $\left(D_{1}, d_{1}\right), \ldots,\left(D_{s}, d_{s}\right)$ are distinct elements of $V\left(\mathcal{G}^{(r)}(q, s)\right)$, then the system of $s$ equations

$$
N\left(D_{j}+X\right)=d_{j} x, \quad 1 \leq j \leq s
$$

has at most $(s-1)$ ! solutions $(X, x) \in G F\left(q^{s-1}\right) \times G F(q)^{*}$.

Theorem 4.2. Let $r \geq 2, s \geq 3$ be fixed positive integers. Let $\mathcal{H}$ be the complete $r$-partite $r$-graph $\left.K^{(r)}(1, \ldots, 1, s, t)\right)$ consisting of $r-2$ sets of size 1 , one set of size $s$, and one set of size $t=(s-1) !+1$. Then $\mathcal{G}^{(r)}(q, s)$ is $\mathcal{H}$-free. Moreover,

$$
\operatorname{ex}(n, \mathcal{H})=\Theta\left(n^{r-1 / s}\right)
$$

and

$$
\operatorname{ex}\left(n, K^{(r)}(1, \ldots, 1,3,3)\right) \sim \frac{n^{r-1 / 3}}{r !} .
$$

Proof. We first show that $\mathcal{G}^{(r)}(q, s)$ is $\mathcal{H}$-free. Then (9) and the fact that for sufficiently large $n$, there is a prime between $n$ and $n+o(n)$ (see [16]) together yield the lower bounds in (10) and (11). Suppose that $\left(A_{i}, a_{i}\right), 1 \leq i \leq r-2,\left(B_{j}, b_{j}\right), 1 \leq j \leq s,\left(C_{k}, c_{k}\right), 1 \leq k \leq t$, form the vertex set of a copy of $\mathcal{H}$ in $\mathcal{G}^{(r)}(q, s)$, where the $\left(A_{i}, a_{i}\right)$ form the parts of size 1 , $\left\{\left(B_{j}, b_{j}\right)\right\}$ forms the part of size $s$, and $\left\{\left(C_{k}, c_{k}\right)\right\}$ forms the part of size $t$. For $1 \leq j \leq s$, set

$$
P_{j}=\left(\sum_{i=1}^{r-2} A_{i}\right)+B_{j} \quad \text { and } \quad p_{j}=\left(\prod_{i=1}^{r-2} a_{i}\right) b_{j} .
$$

Note that $\left(P_{j}, p_{j}\right) \neq\left(P_{j^{\prime}}, p_{j^{\prime}}\right)$ for $j \neq j^{\prime}$, since otherwise $\left(B_{j}, b_{j}\right)=\left(B_{j^{\prime}}, b_{j^{\prime}}\right)$. The edges forming the copy of $\mathcal{H}$ yield, for $1 \leq j \leq s, 1 \leq k \leq t$,

$$
N\left(P_{j}+C_{k}\right)=p_{j} c_{k}
$$

where $P_{j}+C_{k} \neq 0$ for all $j, k$. But Lemma 4.1 implies that such a system can have at most $t-1$ solutions $\left(C_{k}, c_{k}\right)$. 
For the upper bounds in (10), (11), we proceed by induction on $r$. The case $r=2$ is proved in [18] for $s>3$ and in [14] for $s=3$. The inductive step follows immediately by considering the $(r-1)$-graph induced by the neighborhood of a vertex of maximum degree.

\section{$5 \quad$ Proof of Theorem 1.6}

Let $\mathcal{F}$ be an $r$-graph with $v$ vertices and $e>0$ edges. An easy application of the probabilistic deletion method yields $\operatorname{ex}(n, \mathcal{F})>c n^{\alpha}$, where $\alpha=r-(v-r) /(e-1)$ and $c$ is independent of $n$. This yields $\operatorname{ex}\left(n, K^{(3)}(2,2,2)\right)>c n^{18 / 7}$. The exponent $18 / 7$ has recently been improved to $13 / 5$ by Gunderson, Rödl, and Sidorenko [15] but their construction is not explicit.

The previous best known lower bound $\operatorname{ex}\left(n, K^{(3)}(2,2,3)\right)>c n^{29 / 11}$ is also obtained by the probabilistic method. We improve the exponent $29 / 11$ to $8 / 3$ by proving Theorem 1.6 using the Norm hypergraphs described in the previous section. Thus our construction is explicit, and is the first construction giving better bounds than the probabilistic method for the Turán number of $K^{(3)}(i, j, k)$ when $2 \leq i \leq j \leq k$.

In the following proof we write $[m]$ for $\{1, \ldots, m\}$.

Proof of Theorem 1.6: Let $q$ be an odd prime power. Recall that $\mathcal{G}^{(3)}(q, 3)$ has $n=$ $(1+o(1)) q^{3}$ vertices and by (9) it has $(1 / 6+o(1)) q^{8}=(1 / 6+o(1)) n^{8 / 3}$ edges. We will show that $\mathcal{G}^{(3)}(q, 3)$ contains no copy of $K^{(3)}(2,2,3)$. Then the fact that for sufficiently large $n$, there is an odd prime between $n$ and $n+o(n)$ (see [16]) yields the lower bound claimed.

Suppose that $\left\{\left(A_{i}, a_{i}\right)\right\},\left\{\left(B_{j}, b_{j}\right)\right\},\left\{\left(X_{k}, x_{k}\right)\right\}, i, j \in[2], k \in[3]$ forms a copy $\mathcal{F}$ of $K^{(3)}(2,2,3)$, with $\left\{\left(A_{i}, a_{i}\right)\right\}$ and $\left\{\left(B_{j}, b_{j}\right)\right\}$ forming the two parts of size two, and $\left\{\left(X_{k}, x_{k}\right)\right\}$ forming the part of size three. Let $D_{i j}=A_{i}+B_{j}$ and $d_{i j}=a_{i} b_{j}$. Then the edges of $\mathcal{F}$ yield

$$
N\left(D_{i j}+X_{k}\right)=d_{i j} x_{k}
$$

for $i, j \in[2]$ and $k \in[3]$.

If the set $S=\left\{\left(D_{i j}, d_{i j}\right): i, j \in[2]\right\}$ has at least three distinct elements, then Lemma 4.1 with $r=s=3$ implies that (12) cannot hold for each $k \in[3]$. Hence we may assume that $|S| \leq 2$.

If $\left(D_{i 1}, d_{i 1}\right)=\left(D_{i 2}, d_{i 2}\right)$, then $A_{i}+B_{1}=A_{i}+B_{2}$ and $a_{i} b_{1}=a_{i} b_{2}$. This implies that $\left(B_{1}, b_{1}\right)=\left(B_{2}, b_{2}\right)$, a contradiction. Hence we may assume that $\left(D_{i 1}, d_{i 1}\right) \neq\left(D_{i 2}, d_{i 2}\right)$ and by 
symmetry, that $\left(D_{1 j}, d_{1 j}\right) \neq\left(D_{2 j}, d_{2 j}\right)$. Since $|S| \leq 2$, we obtain $\left(D_{11}, d_{11}\right)=\left(D_{22}, d_{22}\right)$ and $\left(D_{12}, d_{12}\right)=\left(D_{21}, d_{21}\right)$. Consequently,

$$
\begin{aligned}
& A_{1}+B_{1}=A_{2}+B_{2} \\
& A_{1}+B_{2}=A_{2}+B_{1}
\end{aligned}
$$

Together these equations yield $2\left(B_{1}-B_{2}\right)=0$, and since $q$ is an odd prime power, this implies that $B_{1}=B_{2}$. The two edges $\left\{\left(A_{1}, a_{1}\right),\left(B_{1}, b_{1}\right),\left(X_{k}, x_{k}\right)\right\}$ and $\left\{\left(A_{1}, a_{1}\right),\left(B_{2}, b_{2}\right),\left(X_{k}, x_{k}\right)\right\}$ yield

$$
a_{1} b_{1} x_{k}=N\left(A_{1}+B_{1}+X_{k}\right)=N\left(A_{1}+B_{2}+X_{k}\right)=a_{1} b_{2} x_{k} .
$$

Consequently $b_{1}=b_{2}$ which provides the contradiction $\left(B_{1}, b_{1}\right)=\left(B_{2}, b_{2}\right)$. This completes the proof.

\section{Concluding remarks and open problems}

- Let $\mathcal{H}=\mathcal{H}(3,2)$ be the 3-graph with $V(\mathcal{H})=\left\{x_{1}, x_{2}, x_{3}, y_{1}, y_{2}\right\}$ and the six edges $\left\{x_{i}, x_{j}, y_{k}\right\}, 1 \leq i, j \leq 3, k=1,2$. Using the proof technique of Theorem 1.4, it follows easily that

$$
\operatorname{ex}\left(n,\left\{C_{4}^{(3)}, K^{(3)}(1,2,3), \mathcal{H}\right\}\right) \leq \frac{5}{6}\left(\begin{array}{l}
n \\
2
\end{array}\right)
$$

We know good bounds for $\operatorname{ex}\left(n, C_{4}^{(3)}\right)$ (Theorem 1.2) and ex $\left(n, K^{(3)}(1,2,3)\right.$ ) (Theorem 3.1), so it seems natural to ask for the asymptotics of $\operatorname{ex}(n, \mathcal{H})$.

- It may be useful to impose some local structure to the hypothesis of Conjecture 1.3. The codegree of a pair of vertices $u, v$ is the number of edges containing them both. For a hypergraph $\mathcal{G}$, let $c(\mathcal{G})$ be the minimum codegree, taken over all pairs of vertices. An easy count shows that if $\mathcal{G}$ is a 3 -graph, then $c(\mathcal{G}) \geq 3$ implies that $|E(\mathcal{G})| \geq\left(\begin{array}{l}n \\ 2\end{array}\right)$. One can ask what minimum value of $c(\mathcal{G})$ forces a copy of $C_{4}^{(3)}$. Theorem 1.2 implies that $c(\mathcal{G})=11$ suffices, and if Conjecture 1.3 holds for $r=3$, then $c(\mathcal{G})=4$ should suffice. Note that the examples in $(1)$ show that $c(\mathcal{G})=3$ does not suffice.

- The $r$-partite $r$-graphs considered in Sections 3 and 4 bear great resemblance to bipartite graphs, since all but two of their parts have size 1 (indeed, this was the feature we exploited to reduce our proofs to known results for graphs). We believe that our lower bound for 
$\operatorname{ex}\left(n, K^{(3)}(2,2,3)\right)$ (Theorem 1.6), however, provides real evidence that these constructions are nontrivial hypergraph constructions. We feel that they will be further exploited to attack Conjecture 1.5.

\section{Acknowledgments}

I wish to thank Prasad Tetali for suggesting the study of $\operatorname{ex}\left(n, K^{(3)}(1,2,4)\right)$, Brendan Nagle for helpful discussions regarding Theorem 3.1, Zoltán Füredi for very useful comments on an earlier draft of this paper, Vojta Rödl for helpful discussions about Theorem 1.6, and a Referee for suggesting the generalization presented in Theorem 2.3.

\section{References}

[1] A. Andreev, On a family of Boolean matrices, Vestnik Mosk. Univ. Ser. 1 (mat.-mech) 41 (1986), 97-100 (in Russian), English translation: Moscow Univ. Math. Bull. 41 (1986), 79-82.

[2] N. Alon, L. Rónyai and T. Szabó, Norm-graphs: variations and applications, J. Combin. Theory Ser. B, 76, no. 2, (1999) 280-290.

[3] N. Alon and J. Spencer, The Probabilistic Method, Wiley, New York, (1992).

[4] P. Erdős, Problems and results in combinatorial analysis, Proc. of the Eighth Southeastern Conf. on Combinatorics, Graph Theory and Computing, Baton Rouge 1977, Louisiana State Univ., Congr. Numerantium XIX, 3-12.

[5] B. Bollobás, Extremal Graph Theory, Academic Press, Inc. Ltd. (1978).

[6] W. G. Brown, On graphs that do not contain a Thomsen graph, Canad. Math. Bull., 9, (1966) 281-289.

[7] K. Culík, Teilweise Lösung eines verallgemeinerten Problem von K. Zarankiewicz, Ann. Soc. Polon. Math. 3 (1956), 165-168. 
[8] P. Erdős, On sequences of integers no one of which divides the product of two others and some related problems, Izv. Naustno-Issl. Inst. Mat. i Meh. Tomsk 2 (1938), 74-82, (Zbl 20, 5.)

[9] P. Erdős, On extremal problems of graphs and generalized graphs, Israel J. Math, 2, (1964) 183-190.

[10] P. Erdős, A. Rényi and V. T. Sós, On a problem of graph theory, Studia Sci. Math. Hungar., 1 (1966), 215-235.

[11] P. Erdős and J. Spencer, Probabilistic Methods in Combinatorics, Academic Press, London-New York, Akadémiai Kiadó, Budapest, (1974).

[12] Z. Füredi, Hypergraphs in which all disjoint pairs have distinct unions, Combinatorica, 4, (2-3), (1984), 161-168.

[13] Z. Füredi, New asymptotics for bipartite Turán numbers, J. Combin. Theory Ser. A, 75 (1996), no. 1, 144-144

[14] Z. Füredi, An upper bound on Zarankiewicz' problem, Combin. Probab. Comput. 5 (1996), no. 1, 29-33.

[15] D. Gunderson, V. Rödl, A. Sidorenko, Extremal Problems for Sets Forming Boolean Algebras and Complete Partite Hypergraphs, J. Combinatorial Theory Ser. A 88 (1999), $342-367$.

[16] M. N. Huxley and H. Iwaniec, Bombieri's theorem in short intervals, Mathematika, 22, (1975), 188-194.

[17] J. Kollár, L. Rónyai and T. Szabó, Norm-Graphs and Bipartite Turán Numbers, Combinatorica, 16, (3), (1996), 399-406.

[18] T. Kővári, V. T. Sós and P. Turán, On a problem of K. Zarankiewicz, Colloq. Math., 3 (1954), 50-57.

[19] S. Roman, A problem of Zarankiewicz, J. Combinatorial Theory Ser. A 18 (1975), 187198. 
[20] D. K. Ray-Chaudhuri and R. M. Wilson, The existence of resolvable block designs, in Survey of combinatorial theory (Proc. Internat. Sympos., Colorado State Univ., Fort Collins, Colo., 1971), (North-Holland, Amsterdam, 1973), 361-375. 